\title{
Política pública nacional de salud bucodental del Paraguay 2009-2018*
}

\author{
National Oral Health Policy of Paraguay, 2009-2018
}

National Oral Health Policy of Paraguay, 2009-2018

Fecha de recepción: 04-10-2018 | Fecha de aceptación: 13-01-2019

\begin{abstract}
Cristina Raquel Caballero García
Universidad Autónoma de Asunción, Asunción, Paraguay. criscaballerog37@gmail.com. https://orcid.org/0000-0003-1667-941X
\end{abstract}

\section{CONCEPCIÓN ELIZABETH GIMÉNEZ DE CHAMORRO}

Universidad Autónoma de Asunción, Asunción, Paraguay. lizy10doc@hotmail.com. https://orcid.org/0000-0003-4554-8662

\section{FranCisCo Flores AlatorRe}

Universidad Autónoma de Asunción, Asunción, Paraguay. pponk@hotmail.com. https://orcid.org/0000-0001-7247-3593

* Artículo de reflexión

Correspondencia: criscaballerog37@gmail.com; lizy10doc@hotmail.com; pponk@ hotmail.com 
doi: $\underline{\text { https://doi.org/10.11144/Javeriana.uo38-80.ppns }}$

Cómo citar: Caballero García CR, Giménez de Chamorro CE, Flores Alatorre JF. Política pública nacional de salud bucodental del Paraguay, 2009-2018. Univ Odontol. 2019 ene-jun; 38(80). https://doi.org/10.11144/Javeriana.uo38-80.ppns

\section{RESUMEN}

Antecedentes: la Política Pública Nacional de Salud Bucodental (SBD) "Por el Derecho de Volver a Sonreír" se inició en el marco de la reforma de salud del año 2008 dirigida hacia un sistema de salud basado en la Atención Primaria de Salud. Objetivo: Analizar el proceso del desarrollo e implementación de la Política Pública Nacional de SBD del Ministerio de Salud Pública y Bienestar Social del Paraguay (MSPBS) 2009-2018. Análisis de la política: Se expone la experiencia del Paraguay utilizando el marco de referencia propuesto por la Organización Mundial de la Salud para la redacción de políticas de SBD. Se describe el proceso dinámico de formulación de la política que incluye: el contexto del Sistema Nacional de Salud, la identificación de la necesidad, las estrategias de intervención implementadas, los pilares que la sustentaron, su evaluación, desafíos y logros. Conclusiones: El establecimiento de la Política Pública Nacional de SBD del MSPBS 2009-2018, logró posicionar a la SBD en la agenda pública del país ubicándola en un sitial de privilegio, nunca antes logrado dentro de la Institución. Su consolidación requirió de una profunda restructuración del programa, así como la implementación de diversas líneas de acción, cuyas evaluaciones, permitieron visualizar un fortalecimiento en la prestación de los servicios y un impacto positivo en la SBD de la población. 


\section{Palabras clave}

abogacía; evaluación de la política; formulación de la política; implementación de la política; política pública de salud bucodental; programas de salud bucodental; Paraguay

\section{Áreas temáticas}

odontología; políticas públicas; salud bucal

\section{ABSTRACT}

Background: The National Public Policy of Oral Health "For the Right to Smile Back" was initiated within the framework of the health reform of 2008 directed towards a health system based on Primary Health Care. Purpose: To analyze the process of development and implementation of the National Public Policy of Oral Health of the Ministry of Public Health and Social Welfare of Paraguay (MPHSW) 2009-2018. Policy analysis: The experience of Paraguay is exposed using the frame of reference proposed by the World Health Organization for the drafting of SBD policies. The dynamic process of formulating the policy is described, which includes: the context of the National Health System, the identification of the need, the intervention strategies implemented, the pillars that supported it, its evaluation, challenges and achievements. Conclusions: The establishment of the National Oral Health Public Policy of the MSPBS 2009-2018, managed to position the oral health in the public agenda of the country placing it in a privileged seat, never before achieved within the Institution. Its consolidation required a deep restructuring of the program, as well as the implementation of various lines of action, whose evaluations allowed to 
visualize a strengthening in the provision of services and a positive impact on the oral health of the population.

\title{
Keywords
}

advocacy; evaluation of the policy; policy formulation; implementation of the policy; public oral health policy; oral health programs; Paraguay

\section{Thematic fields}

dentistry; public policy; oral health

\begin{abstract}
ABSTRATO
Antecedentes: A Política Pública Nacional de Saúde Oral "Pelo Direito ao Sorriso de Volta" foi iniciada no marco da reforma de saúde de 2008, direcionada a um sistema de saúde baseado na Atenção Primária à Saúde. Objetivo: Analisar o processo de desenvolvimento e implementação da Política Pública Nacional de Saúde Bucal do Ministério da Saúde Pública e do Bem-Estar Social do Paraguai (MPHSW) 2009-2018. Análise de políticas: A experiência do Paraguai é exposta usando o quadro de referência proposto pela Organização Mundial de Saúde para a elaboração de políticas de SBD. Descreve-se o processo dinâmico de formulação da política, que inclui: o contexto do Sistema Único de Saúde, a identificação da necessidade, as estratégias de intervenção implementadas, os pilares que a apoiaram, sua avaliação, desafios e conquistas. Conclusões: O estabelecimento da Política Pública Nacional de Saúde Bucal do MSPBS 2009-2018, conseguiu posicionar a saúde bucal na agenda pública do país, colocando-a em um assento privilegiado, nunca antes alcançado dentro da Instituição. Sua consolidação exigiu uma profunda reestruturação do
\end{abstract}


programa, bem como a implementação de várias linhas de ação, cujas avaliações permitiram visualizar um fortalecimento na prestação de serviços e um impacto positivo na saúde bucal da população.

\section{Palavras-chave}

advocacia; avaliação da política; formulação de políticas; implementação da política; política pública de saúde bucal; programas de saúde bucal; Paraguai

\section{Campos temáticos}

odontologia; política pública; saúde bucal

\section{INTRODUCCIÓN}

Por mucho tiempo, las políticas públicas de salud adoptadas por el Paraguay se mostraron ineficientes para responder a las necesidades bucodentales esenciales de la población $(1,2)$. Ese panorama ha caracterizado a muchos países, en especial, a los de bajos y medianos ingresos $(3,4)$. Para la Organización Mundial de la Salud (OMS), una de las principales barreras para la mejora de la salud oral, en la mayoría de los países, ha sido la ausencia de una declaración clara de políticas de salud bucodental (SBD) para orientar sus acciones (5).

Benzian y colaboradores (4), estudiaron las razones de la mencionada negligencia política utilizando el marco para el análisis de generación de prioridades en salud propuesto por Shiffman y Smith (6). Concluyeron que la baja prioridad política para la SBD a nivel mundial era el resultado 
de un conjunto de cuestiones complejas, profundamente enraizadas en el sector que incluye a sus actores clave y sus competencias, a la falta de coherencia y coalescencia; así como a la falta de acuerdos sobre el problema, su descripción y posibles soluciones (4).

Ante dicho contexto ¿cuál es el camino que deben recorrer los países para formular políticas capaces de garantizar la adecuada planificación, gestión y evaluación de programas de SBD? Según Reich (7), los defensores de la reforma de las políticas de salud deben trabajar sobre los tres pilares que sustentan su desarrollo: la voluntad política, el análisis político y las estrategias políticas.

El objetivo de este artículo de reflexión es analizar, con base en documentos gubernamentales e indicadores estadísticos, la experiencia en la formulación, implementación y evaluación de la Política Pública Nacional de SBD del Ministerio de Salud Pública y Bienestar Social del Paraguay (MSPBS). Se realiza la descripción del proceso de desarrollo de la política utilizando el marco de referencia propuesto por la OMS (5). En primer lugar se presenta el contexto del Sistema Nacional de Salud. Posteriormente se expone como se identificó la necesidad de la política pública y más adelante se detalla su formulación, los pilares que la sustentaron, su evaluación, desafíos y logros. Se espera que la experiencia presentada aporte al análisis de la situación de las políticas de salud bucal en la región de las américas y contribuya a promover políticas públicas integrales que garanticen el derecho a la salud.

\section{PROCESO DEL DESARROLLO DE LA POLÍTICA PÚBLICA NACIONAL DE SALUD BUCODENTAL}




\section{Contexto del Sistema Nacional de Salud del Paraguay}

El Sistema Nacional de Salud (SNS), establecido por la Ley 1032/1996 (8), está compuesto por los subsectores público, privado y mixto. El MSPBS desarrolla las funciones de rectoría, provisión y financiamiento, mientras que el Instituto de Previsión Social y el sector privado cumplen con las funciones de aseguramiento, provisión y financiamiento (2,9-12). Desde la década de los noventa del pasado siglo, el SNS ha estado orientado hacia un modelo más funcional y desde el año 2008 hacia un sistema de salud basado en la Atención Primaria de la Salud $(2,10)$.

En la práctica, el SNS presenta alta segmentación de proveedores y carencia de articulación entre los subsectores que lo componen. Trabaja en forma descoordinada, fragmentada, con diversas modalidades de regulación, financiamiento, afiliación y provisión (2,9-12). Características todas que facilitan las desigualdades sociales en salud (12).

En el Paraguay, la Constitución Nacional de 1992 en su artículo 68, establece que el Estado protegerá y promoverá la salud como un derecho fundamental de la persona y en interés de la comunidad (8). En septiembre del 2015, se aprobó la Ley de Salud Indígena, elaborada en consulta con los pueblos indígenas. Sin embargo, este derecho a la salud proclamado en la Constitución se contrapone con los datos sobre aseguramiento y el creciente gasto de bolsillo $(60 \%)$ en salud que se presenta en el país (13).

A partir de 2001 y con el objetivo de disminuir las barreras económicas de acceso a los servicios, el MSPBS, inició la eliminación progresiva de los aranceles cobrados por la prestación de servicios 
$(2,13,14)$. En dicho contexto, por Resolución Ministerial N. ${ }^{\circ}$ 568/2009, se dispone la gratuidad universal de las consultas y procedimientos odontológicos, asumiendo el desafío de garantizar la provisión de servicios (15). A pesar de los logros, aún se requiere continuar con la creación de Unidades de Salud de la Familia (USF) e implementar otras estrategias, a modo de seguir avanzando en cuanto al acceso y cobertura, especialmente en los grupos más vulnerables (13).

\section{IDENTIFICACIÓN DE LA NECESIDAD DE UNA POLÍTICA PÚBLICA NACIONAL DE SALUD BUCODENTAL}

\section{Análisis y diagnóstico situacional del programa de salud bucodental}

En el año 2009, la Dirección de Salud Bucodental (DSBD) del MSPBS realizó un diagnóstico situacional de los programas de SBD y de los servicios de atención odontológica a nivel país, donde también se analizaron las principales barreras que afectaban la ejecución de programas de promoción, de curación y de rehabilitación en dicha área. Los resultados evidenciaron que el programa era completamente ignorado, sub valorado y que existía una histórica barrera de acceso debido a la no gratuidad de la atención odontológica en los servicios públicos (2).

Lo anterior, fue corroborado por una investigación realizada por Caballero-García (16), donde se realizaron entrevistas a actores clave, observación participante, revisión de fuentes secundarias y de documentos del programa de SBD del MSPBS. Se encontró que, por décadas, en el Paraguay, la SBD no había figurado como prioridad en la agenda del gobierno y que no existían estrategias definidas para la provisión de los servicios a la población (16). 
Se pudo corroborar que a finales de la década de los 80', el programa de SBD, manejaba un reducido presupuesto, pero éste se perdió al inicio de la década de los 90', con la justificación de que el nivel era normativo y técnico (16). Las fluctuaciones en el financiamiento no permitían planificar acciones concretas para visualizar cambios sustanciales en la elevada prevalencia de las principales afecciones bucodentales $(2,16)$.

Se estancó el desarrollo de los programas lo que se vio reflejado en la baja demanda de servicios por parte de la población que, además, tenía reducidas expectativas de encontrar solución a sus necesidades en su proceso de búsqueda de la atención. La prevalencia de las extracciones y tratamientos de urgencias era mucho mayor que los tratamientos conservadores y las rehabilitaciones $(2,16)$.

Se identificó una débil comunicación básica entre la DSBD y las Regiones Sanitarias (R.S.) y la desarticulación de estas instancias con la Dirección General de Servicios de Salud. Así mismo se detectó falta de representatividad y nula participación social y comunitaria en los Consejos de Salud y un sistema de información poco desarrollado $(2,16)$.

\section{Antecedentes de la situación de salud bucodental}

En el Paraguay, los resultados de las evaluaciones realizadas por el Programa Regional de SBD de la Organización Panamericana de la Salud (OPS) $(17,18)$ y de la Encuesta Nacional de Salud Oral 2008 (ENSO 2008) (1), mostraron un avance lento con relación a la disminución de la prevalencia de la caries dental, manteniéndose, desde el año 1999 hasta el 2008, en un nivel de severidad moderado, según los criterios de la OMS $(1,17,18)$. 
La ENSO 2008 (1) aplicada a la población escolar de 5-6, 12 y 15 años de edad, reportó que la prevalencia de la enfermedad presentaba cifras que sobrepasaban las metas establecidas por la OMS y la Federación Dental Internacional (FDI) para el año 2000 de lograr que el $50 \%$ de los niños de 5-6 años se encuentren libres de caries (19). Del mismo modo, el índice de dientes cariados, perdidos y obturado (CPO-D) en niños de 12 años, sobre todo cuando se examinaron los datos por R.S., seguía elevado (1).

La caries dental afectaba a alrededor del $80 \%$ de la población infantil y adolescente encuestada $(\mathrm{n}=2589)$. El promedio global del índice de dientes cariados, a extraer y obturados (ceo-d) fue de 4,78 (3,99 para los 5 años y 5,57 para los 6 años). El principal componente fue el elemento cariados con un $96 \%$, seguido por el de obturados con $1,06 \%$ y por el componente a extraer con $1,6 \%$. La experiencia de caries fue del $81 \%(72,3 \%$ para el grupo de 5 años y $90 \%$ para el grupo de 6 años) y la presencia de caries sin tratar del $80 \%$ (1).

Para la dentición permanente la experiencia de caries global fue del 78 \% (75,6 \% a los 12 años y $81,5 \%$ para los 15 años) y la caries sin tratar fue del $68 \%$. El promedio del CPO-D fue de 0,22 para los 6 años, 2,79 para los 12 años y 4,34 para los 15 años. También se presentó como principal componente el elemento caries con un $78 \%$, seguido por los componentes perdidos y obturados con $10,88 \%(1)$.

Según el indicador urgencia de la atención, en todas las R.S. el $43 \%$ de la población de estudio necesitaba tratamiento urgente debido a extensas cavidades, dolor o infección. Con relación al 
Índice Periodontal Comunitario en las edades de 12 y 15 años, el $39 \%$ presentó sangrado gingival, el 6,7 \% presentó cálculos supra o subgingival y el $55 \%$ tenía tejidos periodontales sanos (1).

\section{FORMULACIÓN DE LA POLÍTICA PÚBLICA NACIONAL DE SALUD BUCODENTAL}

\section{Preámbulo}

Desde una perspectiva integral, la salud bucodental, al igual que la salud general, es el resultado de una serie de determinantes sociales como; el estilo de vida, el ingreso económico, la educación, la alimentación, el acceso a los servicios de salud, la cultura y las políticas públicas, entre otros $(20,21)$. Lo anterior implica que para lograr mejoras equitativas y sostenibles es necesaria una visión holística de la salud bucodental, que vaya más allá de los factores de riesgos individuales y que, además, contemple el trabajo coordinado y articulado de múltiples sectores y actores (20-22).

\section{Principios que rigen el cuidado de la salud bucodental}

La Política Pública Nacional de SBD del MSPBS del Paraguay "Por el Derecho de Volver a Sonreir" 2009-2018 se fundamentó en los principios ya consagrados en la Constitución Nacional de 1991 en su artículo 68 (8) y en las regulaciones relacionadas. Buscó adoptar un enfoque integral de la SBD que contemple su conexión con la salud general, el ciclo de vida de las personas, el abordaje de riesgos comunes a otras enfermedades no transmisibles y la articulación del trabajo de todo el equipo de la salud.

Se formuló en el contexto de un cambio de gobierno, que impulsó la denominada: "Políticas Públicas para la Calidad de Vida y Salud con Equidad" (2,9). La mencionada política, trajo 
consigo la gratuidad de los servicios de salud públicos (2009) y el impulso de la estrategia de Atención Primaria de la Salud. Dentro de la cual y, por primera vez, la SBD figuró como prioridad.

\section{Misión}

Mejorar la SBD integral de la población de todo el territorio nacional, interviniendo en las distintas etapas de la vida (2).

\section{Objetivo estratégico institucional}

Mejorar las condiciones de SBD mediante la implementación de programas de promoción de la salud, prevención de enfermedades bucodentales, atención a la salud y rehabilitación integral de acuerdo a las políticas del estado paraguayo (2).

\section{Líneas estratégicas de acción y mecanismos para la implementación}

Para cumplir con el objetivo estratégico institucional se trazaron las diversas líneas de acción que se citan en el (Tabla 1).

TABLA 1

LÍNEAS DE ACCIÓN DE LA DSBD DEL MSPBS

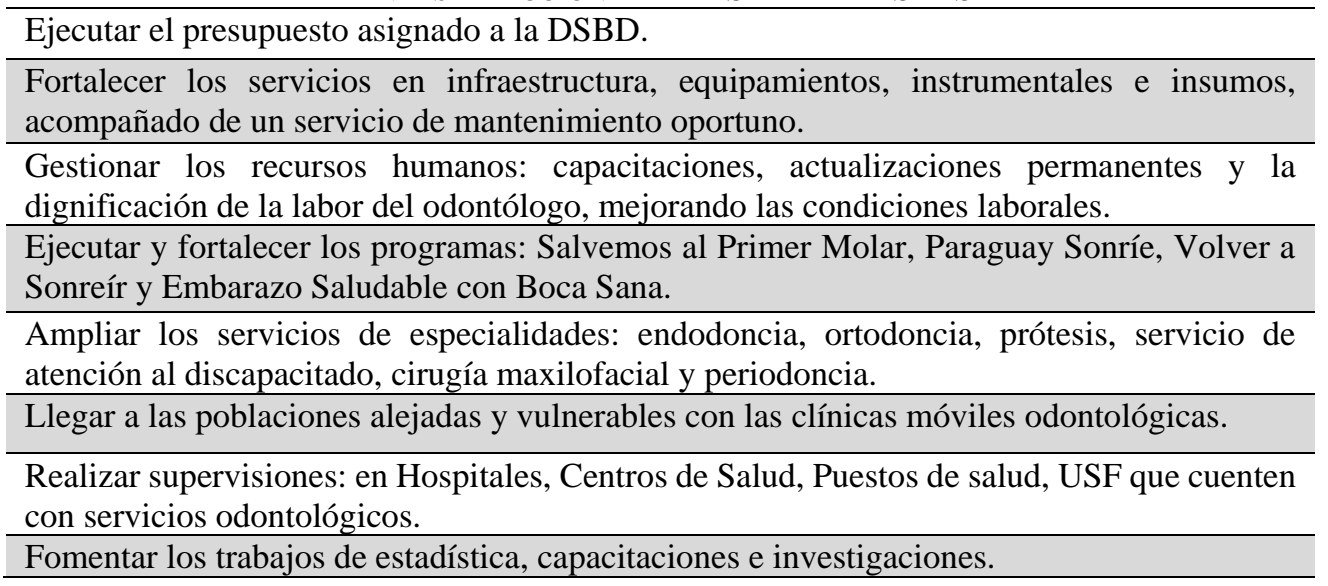


Realizar la fiscalización, habilitación e inspección de los consultorios, las clínicas odontológicas privadas, facultades de odontología y laboratorios de prótesis dentales.

Acreditar las especialidades odontológicas con el Círculo de Odontólogos del Paraguay.

Fuente: Encuesta nacional de salud bucodental del Paraguay, ENSABUD PY 2017 (2).

Las líneas de acción establecidas se enmarcaron en planes de salud bucal, acordes con las políticas de salud del gobierno, aprobadas por Resoluciones Ministeriales que conformaron el marco legal del programa de SBD (Tabla 2). Su implementación requirió de la restructuración del programa de SBD, la identificación de actores clave, el establecimiento de equipos de trabajo en las 18 R. S., la evaluación de las acciones y la abogacía política constante.

TABLA 2

MARCO LEGAL DEL PROGRAMA DE SBD DEL MSPBS

Resolución N. . 300. Por la cual se crea la Dirección de Salud Bucodental (23).

Resolución N. ${ }^{\circ}$ 533. Por la cual se aprueba el Plan de Salud Bucal 2009-2013 (24).

Resolución N. ${ }^{\circ}$ 568. Por la cual se dispone la Gratuidad Universal de las consultas y procedimientos odontológicos (15).

Resolución N. ${ }^{\circ} 86$. Por la cual se aprueba el nuevo organigrama funcional de la Dirección de Salud Bucodental (25).

Resolución No699. Por la cual se aprueba el manual de funciones de la Dirección de Salud Bucodental.

Resolución N. ${ }^{\circ}$ 106. Por la cual se dispone la obligatoriedad de la habilitación de las clínicas y consultorios odontológicos y laboratorios dentales (26).

Resolución N. ${ }^{\circ}$ 421. Por la que se crea el primer Centro de Atención Odontológica a las personas con discapacidad.

Resolución 357. Por la cual se aprueba el Programa "Embarazo Saludable con Boca Sana (27).

Cconvenios interinstitucionales con: el Instituto de Previsión Social, la Sanidad Militar, la Sanidad Policial, las Facultades de Odontología, entre otros.

Cooperaciones internacionales con la OMS/OPS.

Fuente: Encuesta nacional de salud bucodental del Paraguay, ENSABUD PY 2017 (2).

\section{LOGROS DE LA POLÍTICA PÚBLICA NACIONAL DE SALUD BUCODENTAL}


La implementación de la Política Nacional de SBD "Por el Derecho de Volver a Sonreir", desde el año 2009 hasta el año 2018, ha repercutido en importantes avances relacionados a las diferentes líneas de acción, entre ellos (2):

- El logro de una línea presupuestaria propia de salud bucodental (SBD 21 76) que forma parte del presupuesto general del MSPBS. En el año 2015, fue presentada nuevamente en el anteproyecto para el año 2016 y cada año se solicita ampliaciones.

- El diseño y la implementación anual de la Agenda Estratégica de SBD.

- El aumento paulatino en el número de servicios odontológicos en los establecimientos del MSPBS (Tabla 3).

TABLA 3

N. ${ }^{\circ}$ DE SERVICIOS ODONTOLÓGICOS EN ESTABLECIMIENTOS DEL MSPBS

\begin{tabular}{lllllll}
\hline Año & $\mathbf{2 0 0 9}$ & $\mathbf{2 0 1 2}$ & $\mathbf{2 0 1 4}$ & $\mathbf{2 0 1 5}$ & $\mathbf{2 0 1 6}$ & $\mathbf{2 0 1 7}$ \\
\hline $\begin{array}{l}\text { N. }{ }^{\text { }} \text { servicios } \\
\text { odontológicos }\end{array}$ & 242 & 318 & 343 & 350 & 375 & 427 \\
\hline
\end{tabular}

Fuente: Encuesta nacional de salud bucodental del Paraguay, ENSABUD PY 2017 (2).

- El fortalecimiento de los servicios odontológicos existentes, en cuanto a: infraestructura, equipamientos, instrumentales, insumos y mantenimiento. Logro estrechamente relacionado con el aumento del presupuesto del programa en los últimos 11 años.

- La contratación y reorganización de recursos humanos. La cantidad de odontólogos contratados por el MSPBS fue de 676 en el 2009 a 1.449 en diciembre del 2017.

- La planificación y la ejecución de programas:

\section{Programa "Salvemos el Primer Molar"}

Con el objetivo de mejorar, a través de medidas de promoción, prevención y atención odontológica, la salud dental de los niños enrolados en el programa. Fue implementado en el año 2010, en el 
2018 era llevado a cabo en 453 escuelas públicas de las 18 R.S. del Paraguay, alcanzaba a 54.620 escolares y, a través de ellos, al personal escolar, sus familias y sus comunidades. Era desarrollado por 500 profesionales odontólogos y contaba con 80 equipos portátiles distribuidos en las R.S. Los datos sobre la evaluación de este programa están disponibles en: https://new.paho.org/par/index.php?option=com_docman\&task=doc_view\&gid=387\&Itemid=99 $\underline{999}$

\section{Programa "Paraguay Sonríe"}

Con el objetivo de proporcionar rehabilitación protésica dental que permita a los beneficiarios recuperar su apariencia y su función masticatoria además de acceder a una mejor calidad de vida. El programa contaba con un laboratorio central con 4 protesistas y era desarrollado por las clínicas móviles de la DSBD (llegando a lugares muy alejados) y en hospitales que contaban con la especialidad.

La implementación del programa se llevó a cabo mediante alianzas estratégicas con gobernaciones, municipios y facultades causando un impacto social positivo en las comunidades beneficiadas. En el tabla 4 se presentan los datos estadísticos del programa correspondiente a los años 2011-2017.

TABLA 4 DATOS ESTADÍSTICOS DEL PROGRAMA "PARAGUAY SONRÍE"

\begin{tabular}{lcccccccc}
\hline Año & $\mathbf{2 0 1 1}$ & $\mathbf{2 0 1 2}$ & $\mathbf{2 0 1 3}$ & $\mathbf{2 0 1 4}$ & $\mathbf{2 0 1 5}$ & $\mathbf{2 0 1 6}$ & $\mathbf{2 0 1 7}$ & Total \\
\hline $\begin{array}{l}\text { N. }{ }^{\text {de }} \\
\text { prótesis }\end{array}$ & 3.000 & 2.137 & 285 & 404 & 754 & 311 & 1.542 & 8.433 \\
\hline
\end{tabular}

Fuente: Encuesta nacional de salud bucodental del Paraguay, ENSABUD PY 2017 (2).

\section{Programa "Volver a Sonreír"}


Con el objetivo de dar respuesta integral a la alta necesidad no atendida de pacientes con malformaciones estructurales congénitas (fisura de labio y el paladar hendido); se instaló un centro de referencia nacional para la atención de los pacientes como un programa permanente con la intención de mejorar la calidad de vida de los niños y niñas afectados por estas malformaciones, mediante el servicio instalado. Desde el inicio del programa en septiembre del 2009 hasta mayo del 2017 se atendieron a 783 pacientes y se realizaron 132 cirugías.

\section{Programa "Embarazo Saludable con Boca Sana"}

Con el objetivo de contribuir a mejorar la salud y calidad de vida de la mujer embarazada a través de acciones de prevención, promoción y atención integral de la SBD. Desde el inicio del programa en julio del 2016 hasta agosto del 2017 fueron atendidas 43.322 embarazadas en las diversas R.S. del país.

- La atención a las poblaciones vulnerables y de difícil acceso, resultado de la continuidad de las atenciones brindadas por las clínicas móviles (buses odontológicos), realizando procedimientos odontológicos que incluían tratamientos preventivos, curativos, de rehabilitación y actividades de promoción de la SBD. En el tabla 5 se muestran las consultas realizadas en las clínicas móviles durante el periodo 2013-2017.

TABLA 5

N. ${ }^{\circ}$ DE CONSULTAS REALIZADAS EN LAS CLÍNICAS MÓVILES DURANTE EL PERIODO 2013-2017

\begin{tabular}{llllll}
\hline Año & $\mathbf{2 0 1 3}$ & $\mathbf{2 0 1 4}$ & $\mathbf{2 0 1 5}$ & $\mathbf{2 0 1 6}$ & $\mathbf{2 0 1 7}$ \\
\hline $\begin{array}{l}\text { N. }{ }^{*} \text { de } \\
\text { Consultas }\end{array}$ & 17.623 & 13.273 & 13.336 & 10.604 & 10.665 \\
\hline
\end{tabular}

Fuente: Encuesta nacional de salud bucodental del Paraguay, ENSABUD PY 2017 (2). 
También se realizó un trabajo coordinado entre los móviles de las distintas instancias tales como: DSBD, Dirección de Coordinación de R.S., Gobernaciones y otros.

La ampliación y creación de servicios de especialidades (Tabla 6). El Instituto Nacional del Cáncer actualmente posee un departamento de odontología renovado con 3 consultorios totalmente equipados y 7 odontólogos. Con ello, habilitó la especialidad de endodoncia, amplió la cantidad de turnos y aumentó el número de consultas.

TABLA 6

N. ${ }^{\circ}$ DE SERVICIOS CON ESPECIALIDADES

\begin{tabular}{lll}
\hline $\mathbf{2 0 1 5}$ & $\mathbf{2 0 1 6}$ & $\mathbf{2 0 1 7}$ \\
\hline 50 servicios con Rx & 75 servicios con $\mathrm{Rx}$ & 137 servicios con $\mathrm{Rx}$ \\
20 servicios hacen endodoncia & 30 servicios hacen endodoncia & 50 servicios hacen endodoncia \\
4 servicios hacen ortodoncia & 4 servicios hacen ortodoncia & 8 servicios hacen ortodoncia \\
4 servicios hacen prótesis & 4 servicios hacen prótesis & 8 servicios hacen prótesis \\
$4 \quad$ servicios hacen cirugía & 4 servicios hacen cirugía & 4 servicios hacen cirugía \\
maxilofacial & maxilofacial & maxilofacial \\
\hline
\end{tabular}

Fuente: Encuesta nacional de salud bucodental del Paraguay, ENSABUD PY 2017 (2).

- El fortalecimiento de la cooperación técnica con la OPS/OMS con proyectos como: la cooperación técnica entre Paraguay y Uruguay por medio de la cual se impulsó la Técnica de Restauración Atraumática (PRAT); la evaluación del programa "Salvemos al Primer Molar y su estrategia PRAT"; las capacitaciones a los odontólogos para la captura de su productividad en el Sistema para el Área Ambulatorio (SAA); la publicaciones de los resultados de la Evaluación del programa "Salvemos al Primer Molar y la estrategia Prat" (28), de la "Guía del sistema de información en salud" (29), de la "Guía de abordaje integral a la salud bucodental para el equipo de salud" (30), del "Manual del módulo experto para la captura de la iniciativas de promoción de la salud y de prevención de las afecciones bucodentales" (31). Finalmente, la 
cooperación para la realización y publicación de la Encuesta Nacional de Salud Bucodental Paraguay 2017 (ENSABUD PY, 2017) (2).

- La Implementación en la DSBD por la Dirección General de Información Estratégica en Salud del nuevo sistema de recolección de datos estadísticos vía WEB, SAA, para la carga de la productividad de los odontólogos (29).

- El diseño (Equipo Técnico de la DSBD) y el desarrollo (Equipo Técnico de la DGTIC) de la herramienta tecnológica denominada: Módulo Experto para las Iniciativas de Promoción de la Salud y Prevención de Afecciones Bucodentales que fue incorporado al SAA (31). Fue un proyecto de desarrollo tecnológico inédito a nivel regional para documentar el trabajo de promoción de la SBD y prevención de las afecciones bucodentales de los odontólogos en las escuelas y otros contextos de trabajo. Desde su implementación en el año 2015 hasta el 2017 se documentaron 713 iniciativas a nivel nacional. El manual está disponible en: http://www.mspbs.gov.py/bucodental/wp-content/uploads/2013/04/MÓDULO-

\section{EXPERTO.pdf}

- El fortalecimiento de la comunicación y del trabajo articulado entre la DSBD y las 18 R.S. Se realizaban supervisiones, monitoreos mensuales de la productividad a través del SAA y controles de gestión anuales, entre otras actividades.

- La formación y consolidación del equipo técnico de la DSBD. La realización de investigaciones, capacitaciones, jornadas de actualización y encuentros de salud pública nacional e internacional. Actualmente el Paraguay es integrante del Observatorio Iberoamericano de Políticas en SBD y realiza actividades y proyectos en unión regional.

- El aumento del número de consultas y procedimientos en todas las R.S., evidenciando mejoras en el acceso. Tendencia a la disminución de las extracciones a favor de los tratamientos 
curativos (número de restauraciones, inactivaciones, periodoncias, endodoncias, ortodoncia, medicación, etc.), hasta el punto de que, en conjunto, han superado a las extracciones dentarias en la mayoría de las R.S. Sumándose los procedimientos preventivos y la promoción de la SBD.

- Encuesta Nacional de Salud Bucodental (2). Los resultados de la ENSABUD PY 2017, en cuanto a la prevalencia de caries $(63,31 \%)$ para la población general $(\mathrm{n}=2.727)$ indican una disminución aproximada de 16,69 puntos porcentuales con relación a los datos de la ENSO 2008, donde la enfermedad afectaba a alrededor del $80 \%$ de la población encuestada $(\mathrm{n}=2589)$. El cambio en la prevalencia abarcó a todas las edades de interés (5-6,12 y 15 años) $(1,2)$. En cuanto a la experiencia de caries tanto en dientes temporarios como en permanentes, la comparación de los datos obtenidos con relación a los de la ENSO 2008, también indican una disminución del porcentaje de la población que presentó evidencia de haber sufrido, en algún momento de su vida, lesiones cariosas y/o secuelas (2). El promedio del índice ceo-d para el grupo de 5-6 años de edad fue de 3,88, valor inferior a lo reportado en la ENSO 2008 donde fue de $4,78(1,2)$.

El promedio del CPO-D actual para la edad de 5-6 años fue de 0,17, para los 12 años de 2,07 y para los 15 años de 3,14, los que también resultaron inferiores a los expuestos en la ENSO 2008 (1), que fueron de 0,22, 2,79 y 4,34 respectivamente. Cabe resaltar que el CPO-D de 2,07 para el grupo de 12 años (edad para comparaciones internacionales), representa el menor valor logrado para el país. Su disminución ha sido paulatina presentando cifras de 5,90 en el año 1983; 4,02 en 1987; un retroceso en 1996 llegando a 5,08; para continuar disminuyendo en los reportes de los años 1999 y 2008 con cifras de 3,83 y 2,79, respectivamente $(1,2)$. 
Los resultados de la ENSABUD 2017 (2) y de la ENSO 2008 (1), coinciden en colocar al elemento cariados, como el principal componente de los índices ceo-d (86,06\% versus $97,16 \%)$ y CPO-D $(69,46 \%$ versus $78 \%)$. También coinciden en reportar al elemento a extraer como el segundo componente con mayor peso en el índice ceo-d, seguido del elemento obturados que, si bien, presenta un valor superior $(2,48 \%)$ con relación al 2008 (1,06 \%) continúa bajo. A diferencia de lo anterior, presentan desacuerdo con relación a los elementos perdidos y obturados del CPO-D, mientras la ENSO 2008 reporta coincidencia entre ambos elementos con un valor de 10,88\%, en la actual encuesta el elemento obturados $(22,13 \%)$ presenta mayor peso con relación al elemento perdidos $(7,63 \%)(1,2)$.

Este avance hacia la disminución de la enfermedad resulta alentador, más aún, cuando analizado en un panorama Regional, donde las publicaciones más recientes de la OPS indican que la caries dental sigue afectando a casi el $90 \%$, de los habitantes de 5 a 17 años de edad en la Región de Latinoamérica y el Caribe $(3,18,32)$.

El cambio epidemiológico no ha ocurrido de manera uniforme para toda la población, de ahí, que presentar, analizar y entender la dinámica de la distribución de las afecciones a nivel país, más allá de los promedios nacionales, es decisivo para disminuir su prevalencia, mejorar la asignación de recursos, así como para reducir las inequidades en SBD, por lo que merecen ser abordadas (2).

Si bien, la prevalencia de caries de la población general fue del 63,31\%, la mayor prevalencia se presentó en el área rural $(74,58 \%)$ mientras que en el área urbana fue de 57,75\%. Este comportamiento fue el mismo para todas las edades (2). 
La experiencia de caries en dientes temporarios se presentó en mayor porcentaje para el área rural $(44,93 \%)$ con relación al área urbana $(32,91 \%)$. Por el contrario, para los dientes permanentes, la experiencia de caries fue mayor para el área urbana con un 40,16 \% con relación al área rural que presentó un $38,35 \%$ (2).

Con relación al índice ceo-d que presentó un valor general de 2,01, cuando es analizado por área de residencia muestra un valor inferior para el área urbana con un promedio de 1,68 (cariados 85,71 $\%$, extracción indicada $10,11 \%$ y obturados $2,97 \%$ ) con relación a la rural que fue de 2,67 (cariados $85,76 \%$, extracción indicada $12,35 \%$ y obturados $1,64 \%$ ) la cual presentó mayores porcentajes de niños en todos los niveles de severidad de la caries dental (2).

Si bien la información sobre la población indígena en la ENSABUD PY 2017, es considerada exploratoria, muestra claramente la situación de desventaja social por pertenecer a un grupo étnico. Presentando una prevalencia de caries del $80,4 \%$ cifra considerablemente superior al de la población general (urbana-rural) que fue del 63,31\% (2).

En la figura 1 puede observarse el proceso de desarrollo de la Política Pública Nacional de Salud Bucodental MSPBD del Paraguay "Por el Derecho de Volver a Sonreír” 2009-2018 en el marco propuesto por la OMS para la formulación de políticas públicas de salud bucodental (5). 
FIGURA 1

FLUJOGRAMA DEL PROCESO DINÁMICO DE LA POLÍTICA PÚBLICA NACIONAL DE SBD DEL MSPBD DEL PARAGUAY 2009-2018

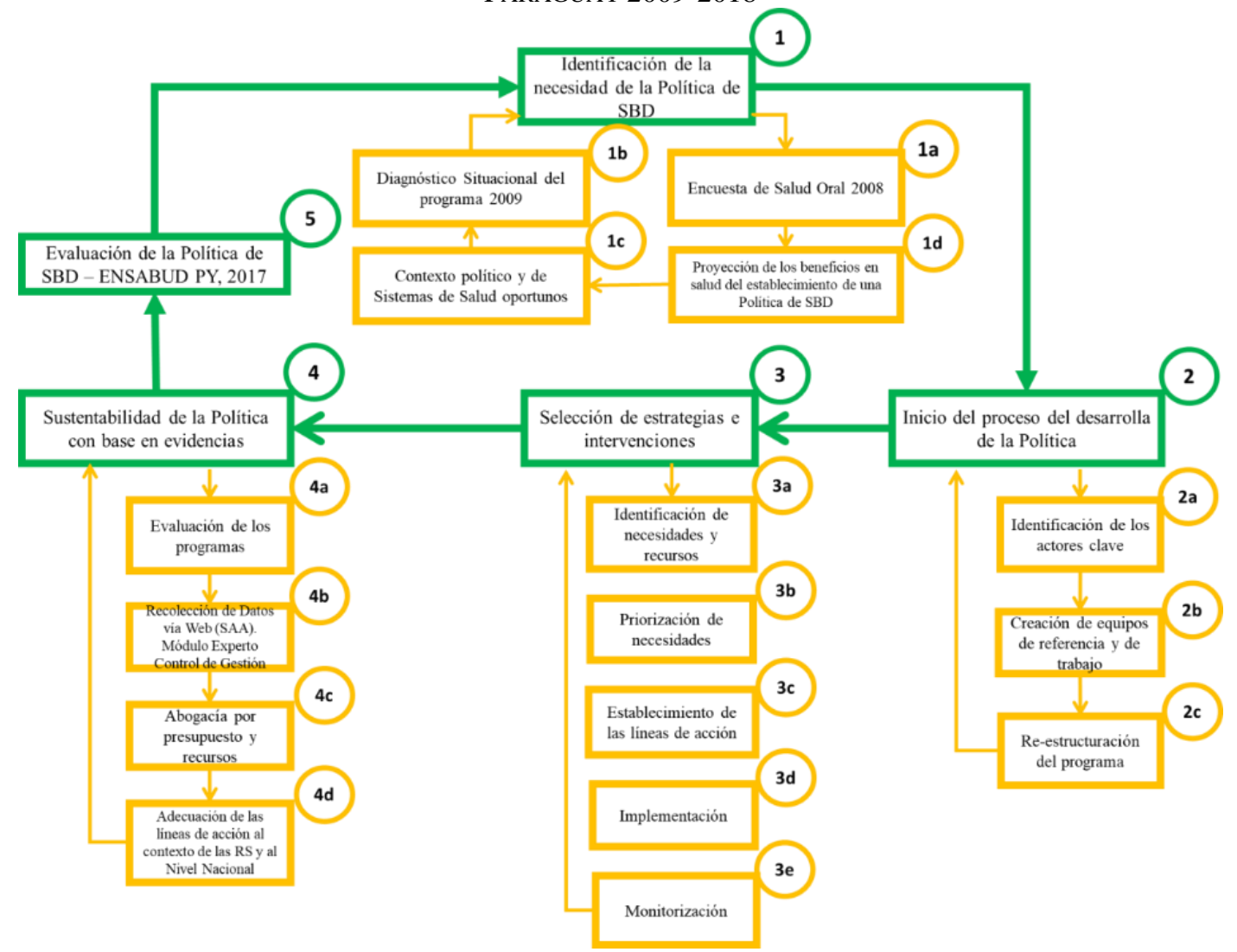

Fuente: Adaptado de WHO. Writing oral health policy: A manual for oral health managers in the WHO African Region 2005 (5).

\section{CONCLUSIONES}

El desarrollo de la Política Pública Nacional de SBD del MSPBD del Paraguay: "Por el Derecho de Volver a Sonreír” se inició aprovechando un contexto político y del sistema de salud, oportuno. Tuvo como base evidencias científicas del diagnóstico de la situación del programa de SBD y la ENSO 2008 realizada en población escolar. 
Su implementación se logró estableciendo instrumentos normativos que significaron profundos cambios estructurales en el programa de SBD del MSPBS y requirieron de constantes análisis y estrategias políticas.

La mencionada política logró consolidarse a través de importantes líneas de acción como: la ejecución del presupuesto asignado a la DSBD; el fortalecimiento de los servicios; la ejecución de programas; la atención a poblaciones vulnerables y la ampliación de los servicios de especialidades, entre otras.

La principal limitación durante el proceso de desarrollo e implementación fue el casi nulo presupuesto inicial asignado al programa de SBD que, si bien, sufrió ampliaciones año tras año, siempre resultó insuficiente. La burocracia de los procedimientos administrativos representó una importante barrera y la actitud negativa o de resistencia de una minoría de odontólogos de realizar su trabajo dentro de las normativas se presentó como un desafío constante.

La sustentabilidad de la política y el logro de una línea presupuestaria requirió de abogacía con autores clave y tuvo su cimiento en las evidencias generadas por la sistematización de las acciones, las evaluaciones de los programas, la carga de la productividad de los profesionales del MSPBS en el SAA y en el Módulo Experto para la carga de Iniciativas de promoción de la SBD y prevención de las afecciones bucodentales. 
Los resultados de la ENSABUD PY 2017, se dan en el marco del desarrollo de la Política Nacional de Salud Bucodental 2009-2018 y sugiere un impacto positivo en la salud bucodental de la población escolar.

Para seguir avanzando en los resultados en SBD es indispensable la continuidad de las acciones y la sostenibilidad de los programas que evidencian efectividad. Del mismo modo, se deben establecer cambios en las políticas que ayuden a reducir el impacto de los problemas de salud oral y sus desigualdades. Se deben enfocar esfuerzos en la identificación y el abordaje de las influencias más amplias de la salud oral como son la familia, la comunidad, el ámbito social y ambiental. Recopilar datos de manera regular y evaluar los modelos de atención para corroborar si son aplicables en diferentes contextos.

\section{AGRADECIMIENTOS}

Por su contribución fundamental para el desarrollo de la Política Nacional de Salud Bucal "Por el Derecho de Volver a Sonreir" 2009-2018 un agradecimiento a las Autoridades Nacionales, a todos los odontólogos del sistema de salud bucal pública, a los equipos de trabajo de la Dirección de Salud Bucodental: Equipo Técnico, de Fiscalización; de las Clínicas Móviles, del laboratorio y Personal Administrativo.

\section{REFERENCIAS}


1. Ministerio de Salud y Bienestar Social Dirección de Salud Bucodental República de Paraguay. Encuesta Nacional Sobre Salud Oral Paraguay 2008:1-98. Asunción. Paraguay OPS/OMS. Año $2009 . \quad$ Disponible en: http://new.paho.org/par/index.php?option=com_docman\&task=doc_details\&gid=73\&Itemid $=239$

2. Ministerio de Salud Pública y Bienestar Social. Encuesta Nacional de Salud Bucodental del Paraguay, ENSABUD PY 2017: Del diagnóstico al diseño y la ejecución de políticas públicas de salud bucodental. Asunción: OPS/OMS 2018. 180 págs. Disponible en: http://portal.mspbs.gov.py/bucodental/wp-content/uploads/2018/09/BUCODENTAL-PY2017_FINAL_AGOSTO-2018.pdf

3. Organización Panamericana de la Salud. Condiciones de salud y sus tendencias En: Salud en las Américas 2007; 2: 143-146. Disponible en: http://www.paho.org/salud-en-las-americas2012/dmdocuments/salud-americas-2007-vol-1.pdf

4. Benzian H, Hobdell M, Holmgren C, Yee R, Monse B, Barnard JT, van Palenstein Helderman W. Political priority of global oral health: an analysis of reasons for international neglect. Int Dent J. 2011; 61(3): 124-130. http://www.doi.org/DOI: 10.1111/j.1875-595X.2011.00028.x.

5. World Health Organization. Writing oral health policy: A manual for oral health managers in the WHO African Region. Regional office for Africa Brazzaville; 2005. Disponible en: http://apps.who.int/iris/bitstream/handle/10665/204462/AFR_ORH_05.1.pdf?sequence=1\&is Allowed=y

6. Shiffman J, Smith S. Generation of political priority for global health initiatives: a framework and case study of maternal mortality. Lancet. 2007 oct 13; 370(9595): 1370-9. http://www.doi.org/ DOI: 10.1016/S0140-6736(07)61579-7 
7. Reich M. The politics of reforming health policies. Promotion and Education. 2009; 9 (4): 138140. Disponible en: content/uploads/sites/480/2012/10/Politics_of_reform.pdf

8. República del Paraguay. Constitución Política de 1992. Asunción. Paraguay. Convención Nacional Constituyente.1992.Disponible en: http://www. constitution.org/cons/paraguay.htm.

9. Organización Panamericana de la Salud. Perfil de los Sistemas de Salud de Paraguay: Monitoreo y análisis de los procesos de cambio y reforma. Washington D.C. EE.UU. OPS 2008: 80 págs. $\quad$ Disponible http://www1.paho.org/hq/dmdocuments/2010/Perfil_Sistema_Salud-Paraguay_2008.pdf

10. Mancuello J. Cabral M. Sistema de Salud de Paraguay. Rev. Salud Pública Parag. 2011; Vol. 1 (1): 13-25. Disponible en: http://www.ins.gov.py/revistas/index.php/rspp/article/view/20/19

11. Ministerio de Salud Pública y Bienestar Social / OPS-OMS Dirección General de Planificación y Evaluación-MSPBS. Sistemas de Salud en Sudamérica: desafíos para universalidad, la integralidad y la equidad. Asunción. Paraguay. OPS-OMS. 2012. Disponible en: sss-paraguay2011.

12. Ministerio de Salud Pública y Bienestar Social del Paraguay/Dirección General de Planificación y Evaluación. Plan estratégico institucional 2013-2018. Asunción. Paraguay. MSPyBS-Paraguay. 2013. Disponible en: https://www.mspbs.gov.py/planificacion/planificacion-estrategica.html

13. Organización Mundial de la Salud. Salud en las Américas. Reporte de país: Paraguay 2017. Washington, D.C. EE.UU. OPS/OMS. 2017. Disponible en: http://www.paho.org/salud-enlas-americas-2017/?page_t_es=informes $\% 20 \mathrm{de} \% 20$ pais/paraguay\&lang=es 
14. Giménez E. Hacia un sistema de salud con garantías. Notas para nuevas políticas de salud en Paraguay. Asunción. Paraguay. Instituto Desarrollo. 2013. ISBN: 978-99967-713-0-9 Disponible en: http://desarrollo.org.py/admin/app/webroot/pdf/publications/12-10-2015-1119-55-343723993.pdf

15. Ministerio de Salud Pública y Bienestar Social del Paraguay. Resolución S.G. N. 568. Asunción. Paraguay. MSPyBS-Paraguay. 2009. Disponible en: http://portal.mspbs.gov.py/bucodental/wp-content/uploads/2013/04/Resolucion-N568.pdf

16. Caballero García CR. Tesis doctoral. Utilización de los servicios odontológicos y calidad de vida: una perspectiva desde la salud bucodental positiva en el Paraguay. Cuernavaca/Morelos. México. Instituto Nacional de Salud Pública/Escuela de Salud Pública de México. 2014. Disponible en: http://www.inspvirtual.mx/banco_tesis/Caballero

17. Pan American Health Organization. Final Report to the W.K. Kellogg Foundation. Project \# 43225. Multiyear Plan for Salt Fluoridation Programs in Region of the Americas (Belize, Nicaragua, Panama, Paraguay and Venezuela). Washington, DC. EE.UU. OPS/OMS. 2000. Disponible en: http://www1.paho.org/hq/dmdocuments/2009/kellogg.pdf

18. Estupiñán S. Promoción de la salud bucodental: el uso de la fluoruración de la salud para prevenir la caries dental. Organización Panamericana de la Salud. Washington DC. EE.UU. 2006. 115 págs. Disponible en: http://new.paho.org/hq/dmdocuments/2009/salt\%20book.pdf

19. Hobdell M. Petersen PE. Clarkson J. Johnson N. Global goals for oral health in the year 2000. FDI/WHO. Int Dent J. 1982; 32: 74-7. Disponible en: http://www.who.int/oral_health/media/en/orh_goals_2020.pdf

20. Commission on Social Determinants Health. Closing the gap in a generation: Health equity through action on the social determinants of health. Final Report of the Commission on Social 
Determinants of Health. Geneva, Switzerland: World Health Organization 2008. Disponible en: http://www.who.int/social_determinants/final_report/csdh_finalreport_2008.pdf

21. Kwan S. Petersen E. Oral health: Equity and Social Determinants. Chap 9 in: Equity, social determinants and public health programmes. Editors Erik Blas and Anand Sivasankara Kurup. WHO 2010; pp. 159-78.

22. Watt RG. From victim blaming to upstream action: Tackling the social determinants of oral health inequalities. Community Dent Oral Epidemiol. 2007; 35: 1-11. 10.1111/j.16000528.2007.00348.x

23. Ministerio de Salud Pública y Bienestar Social del Paraguay. Resolución S.G. N. 300. Asunción. Paraguay. MSPyBS-Paraguay. 1994 Disponible en: http://portal.mspbs.gov.py/bucodental/wp-content/uploads/2013/04/Resolucion-N300.pdf

24. Ministerio de Salud Pública y Bienestar Social del Paraguay. Resolución S.G. N. ${ }^{\circ} 533$. Asunción. Paraguay. MSPyBS-Paraguay. 2009 Disponible en: http://portal.mspbs.gov.py/bucodental/wp-content/uploads/2013/04/Resolucion-N533.pdf

25. Ministerio de Salud Pública y Bienestar Social del Paraguay. Resolución S.G. N. 86. Asunción. Paraguay. MSPyBS-Paraguay. 2013. Disponible en: http://portal.mspbs.gov.py/bucodental/quienes-somos/

26. Ministerio de Salud Pública y Bienestar Social del Paraguay. Resolución S.G. N. 106. Asunción. Paraguay. MSPyBS-Paraguay. 2013. Disponible en: http://portal.mspbs.gov.py/bucodental/wp-content/uploads/2013/04/Resolucion-106.pdf

27. Ministerio de Salud Pública y Bienestar Social del Paraguay. Resolución S.G. N. 357. Asunción. Paraguay. MSPyBS-Paraguay. 2016. Disponible en: 
http://portal.mspbs.gov.py/bucodental/wp-content/uploads/2016/08/Resoluciondise\%C3\%B1o-y-planificaci\%C3\%B3n-del-programa_2016.pdf

28. Caballero-García CR, Flores-Alatorre JF. Evaluación del Programa: "Salvemos al Primer Molar" y la estrategia PRAT del Ministerio de Salud Pública y Bienestar Social del Paraguay. Proyecto de Cooperación Técnica entre países (TCC) Paraguay-Uruguay. Organización Panamericana de la Salud. Asunción. Paraguay. OMS/OPS. 2012.

29. https://www.paho.org/par/index.php?option=com_docman\&view=download\&category_slug =publicaciones-con-contrapartes\&alias=387-evaluacion-del-primer-molar\&Itemid=253

30. Ministerio de Salud Pública y Bienestar Social del Paraguay/Organización Panamericana de la Salud. Guía del sistema de información en salud área ambulatorio para el uso odontológico 2012. Asunción. Paraguay. MSPyBS-Paraguay. 2012. Disponible en: http://portal.mspbs.gov.py/bucodental/wp-content/uploads/2013/04/GUIAbucodental_enero20131.pdf

31. Ministerio de Salud Pública y Bienestar Social del Paraguay/Organización Panamericana de la Salud. Guía de abordaje integral a la salud bucodental para el equipo de salud 2012. Asunción. Paraguay. $\quad 2012 . \quad$ DSPyBS-Paraguay. en: http://portal.mspbs.gov.py/bucodental/wp-content/uploads/2013/04/GuiaAbordaje_enero20131.pdf

32. Ministerio de Salud Pública y Bienestar Social del Paraguay/Organización panamericana de la Salud. Odontología en red para medir el impacto del trabajo en la promoción de la salud bucodental. Manual del módulo experto para las iniciativas de promoción de la salud y prevención de afecciones bucodentales. Asunción. Paraguay. MSPyBS-Paraguay. 2016. ISBN: 
978-99967-36-35-3. Disponible en: http://portal.mspbs.gov.py/bucodental/wpcontent/uploads/2013/04/M\%C3\%93DULO-EXPERTO.pdf

33. Organización Mundial de la Salud. La salud oral de los niños de bajos ingresos: procedimientos para el tratamiento restaurativo atraumático (PRAT). Informe final. ATN/JF-7025-RG. Número de proyecto 091024. Washington DC. EE.UU. OPS/OMS/BID. 2006. Disponible en: http://new.paho.org/hq/dmdocuments/2009/OH-PRAT_mar2009.pdf 